\title{
OPTIMUM USE OF MATERIAL IN BIOLOGICAL ASSAYS
}

\author{
By Warren W. Nicholas
}

\section{ABSTRACT}

It is shown that the procedure often adopted in biological assay to determine the mortality-dosage curve is very wasteful of animals and of time. The available animals are usually divided into equal groups to which are administered various doses which differ in strength by equal steps; the inefficiency is due to the fact that various points on the curve are determined with different degrees of accuracy. A method is described which will determine the curve with uniform accuracy throughout its extent-here the animals are divided into unequal groups, in which the relative numbers are described by a simple mathematical expression.

The case usually encountered in routine biological assay where a single point on the curve is to be determined (that is, corresponding to a biological test for strength of a preparation) is also treated. A method is described which makes the best use of a given number of animals, and permits the results to be expressed quantitatively.

\section{CONTENTS}

I. Introduction _.

II. To determine the entire characteristic curve 80

III. To determine the dose required to produce a given mortality 84

\section{INTRODUCTION}

In biological assay, in order to specify the amount of drug (or other effective agent) required to produce a certain effect (say mortality) in a given organism, it is customary to specify a dosage $\left(D_{o}\right)$ of the agent which produces the "end-point" effect in a certain specified percentage of the organisms in a group sufficiently large in number to reduce the probable errors to a small quantity. The necessity for such a way of specifying dosage is apparent from a consideration of what will here be called after Trevan " the "characteristic curve," which describes the effect of various doses of the effective agent on the organisms. A characteristic curve which is approximated in a great majority of such reactions is shown in Figure 1; here "survival" (that is, fraction of organisms in which the agent did not produce the end-point effect) is plotted as ordinates, and dosage $(D)$ as abscissæ. ${ }^{2}$ It is seen that even doses which are a small fraction of $D_{o}$ can produce

3 J. W. Trevan, Proc. Roy. Soc., B. 101, p. 483; 1927. Trevan's treatment of the errors associated ;with the determination of toxicity closely parallels that in the present paper at many points, but Trevan did not discuss the present applications explicitly.

'It may be pointed out that, if desired, $D$ may represent throughout the present paper the dose per unit susceptibility of an animal instead of the actual dose per animal; that is, corrections for weight, sex, etc., may be made without affecting the results of the paper so long as the meaning of $D$ is consistent throughout the calculations of any one experiment. (For example, if the Dreyer-Walker correction for weight held, the dose administered to an animal might be taken to be $D W .00$ where $W$ is the weight of the animal, and $D$ is the quantity appearing in the equations of the paper.) It is obvious, however, that any statistical analysis such as is given here will be valid whether such corrections are made or not, since the neglect of the corrections will simply superpose another random variation upon the variations already existing; the characteristic curve will, of course, be broadened by the neglect of such corrections. 
the effect in a fraction of the organisms, and that even for doses considerably in excess of $D_{o}$, the effect is not produced in all the organisms. The cause for this large degree of variability in the reactions of the different individuals is not yot ascortained, but in any ovent, this uncertainty as to the underlying cause is no factor in the present discussion.

A great number of organisms is required to determine $D_{o}$ with any considerable degree of certainty, and there has been considerable criticism of the paucity of material used in many assay experimonts. But even when a large amount of biological material (as for example, a large number of animals and correspondingly large amount of drug) has been used, it is often questionable whether the best use has been made of the material. For example, suppose the characteristic curve has been ascertained using, say, eight different values for the dose of poison

$$
1 / 4 D_{o}, 1 / 2 D_{o}, 3 / 4 D_{0} \ldots . . .2 D_{0}
$$

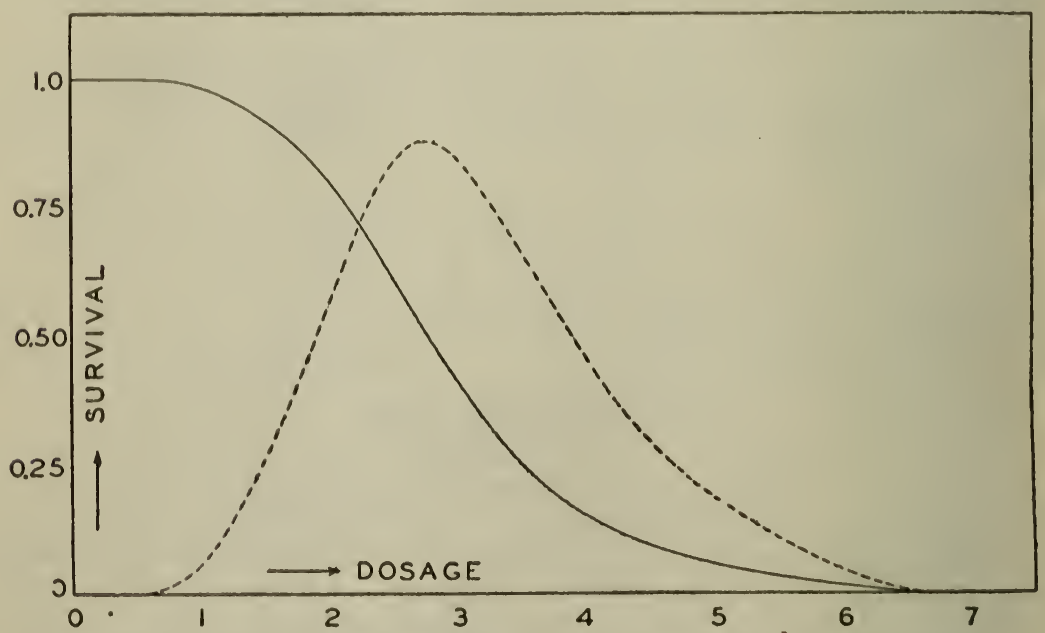

Figure 1.-Typical characteristic curve, with best distribution of counts

Contimus lino "characteristic curve" (see text) whlch is approximnted in a majority of biologicn reactions denling with) mortality (lioto $\mathrm{X}$ rays, mltrn-vlolet light, and various toxic agents, physiobogienl effocts of hormones and vitamlns, and hemolysis due to specific sora. isrokon line, best distribution of individual organisms or counts for determining the characteristic curve with uniform accuracy along its longth.

where $D_{o}$ is in this example the dose required to kill half the animals, and giving to one-eighth of the total number of animals each respective value of the dose. 'Then for the characteristic curve ordinarily obtained it can be shown that with the ordinary use of the data found (that is, without special weighting), more than half ot the animals and poison have been wasted so far as an exact determination of the characteristic curve is concerned, and the experiment could have been done just as accurately with less than half of the animals and poison, or could have been done 1.4 times as accurately using. all the material. ${ }^{3}$

3 The above point is entirel y apart from the consideration that, for a given experimental uncertainty in the dose alministered to an nnimal, tho uncortainty thereby caused in tho mortality is greatest at the stecprst point on the charanterist ic curve. This latter edect is not discussod in the present paper; but a correction for the effect is readily mado ouce the uncertainty in the administered doso is known. 
It will be shown below that, where the dosages are uniformly distributed along the axis of abscissæ, as in the above example, the best use of material is made by dividing the animals into unequal groups (instead of equal, as in the example above), the group containing the largest number being given the dose which will kill half of them and the groups becoming continually smaller for both increasing and decreasing doses. 'The quantitative relation which will be found for the optimum use of material (assuming it is desired to obtain all points on the characteristic curve with equal accuracy) is

$$
\frac{n}{n_{o}}=\frac{s(1-s)}{0.25}
$$

where $n$ is the number of individuals in the group given a dosage for which the fractional (not percentage) survival is $s$, and $n_{0}$ is the number in the group given a dose for which $s$ is 0.50 . It is apparent, from the fact that survival is equal to 1 minus the mortality $(m)$, that the numerator of the fraction on the right-hand side can be written in two other ways, namely, $s m$ and $m(1-m)$. The curve corresponding to this formula, as applied to the characteristic curve shown in Figure 1, is given in the broken curve of Figure $1 .^{4}$

In cases where any number of organisms and any amount of the effective agent are available, it is not necessary to have regard to the optimum use of material. However, even in these cases, it often happens that the administration of the agent or the counting of the affected and surviving organisms is tedious or difficult; in this event, the analysis given below is of as much value as in the experiments in which a saving of material was important, the difference being that here a saving of time and trouble is effected. $\Lambda$ n example of experiments tending toward the latter type are those of Packard on the effects of $\mathrm{X}$ rays on Drosophila eggs. Here a great number of eggs were used in order to obtain high precision in a test of certain quantitative theories for the nature of the biological action of the rays. In fact, the present investigation was suggested by an observation of Packard's that certain points on the characteristic curve seemed to have associated with them a greater uncertainty of determination than others. ${ }^{5}$

The analysis contained in the present paper applies to a great many biological reactions - in fact to every kind in which the independent variable is the quantity or concentration of some effective agent, such as $\mathrm{X}$ rays, ultra-violet light, many toxins and inorganic drugs, hormones, vitamins, specific sera, etc., and the dependent variable is the percentage of cases in which a given end-point effect is produced in a group of individuals, this effect being of the nature of something (such as mortality, hemolysis, production of oestrus, etc.), which can either happen or fail to happen to an individual. The first part of the paper deals with the best distribution of animals

\footnotetext{
- In the past it has sometimes been the practice to mark on the graph the number of animals used for the determination of each point. In the future it will be sufficient to specify the total number of animals used for the entire graph, and to say that they were distributed according to the rclation $m(1-m)$. The dosages used will be evident from the points plotted on the graph.

s Charles Packard, J. Cancer Res., 11, P. 1; 1927. Packard interpreted his observation that the greatest, uncertainties were associatod with mortalitles in the region of 50 per cent as signifying that a greater degree of biological variation was associated with organisms which were susceptible to the range of doses in the vicinity of that which produced a 50 per cent mortality. As will appear below, however, this interpretation can not be accepted without further analysis of the experimental data in terms of the present results.
} 
to use in case the characteristic curve is known approximately, and it is desired to ascertain it more accurately, as has been the case, for example, in Packard's experiments cited above. The second part of the paper deals with the more usual case in biological assay where it is not so important to determine the whole curve, but only one point on it, for example the dose required to produce 50 per cent mortality. The reason for discussing the two parts of the paper in this apparently inverse order is because some of the formulas to be developed in the first part of the paper will be needed in the second part.

\section{TO DETERMINE THE ENTIRE CHARACTERISTIC CURVE}

Any determination of an "optimum use of material" in the present sense implies such a use of material as involves the least error in the final result, as indicated by statistical analysis. In order to make familiar the application of statistics to the present problem, let us first consider a special numerical case. Suppose an individual organism be givén a dose $D_{o}$ of some effective agent, say a poison. If, for example, this dose is that which will kill half of any very large group of similar organisms, we may conclude that the individual which we have selected at random will have an equal chance of dying $(+)$ (that is, that we will obtain a positive effect) and surviving $(-)$. We may represent this state of affairs by the symbolization.

$$
+
$$

giving, in our minds, equal "weights" to both. Suppose next that two individuals, $A$ and $\mathrm{B}$, are each administered $D_{o} ; \mathrm{A}$ has as before equal chances of + and - . Whether $A$ dies or not does not affect the reaction of B, so that there are equal chances, when A dies, of B's dying and surviving; this may be represented by ++ and +- . By similar reasoning we can show that the other two possibilities, -+ and -- , are equal, and also that +- is equal to - Thus we have finally four equally probable possibilities for the effect of $D_{o}$ on two individuals

$$
\begin{aligned}
& ++ \\
& +- \\
& -+ \\
& --
\end{aligned}
$$

The above reasoning may be nicely symbolized and the results extended to any number of individuals as follows:
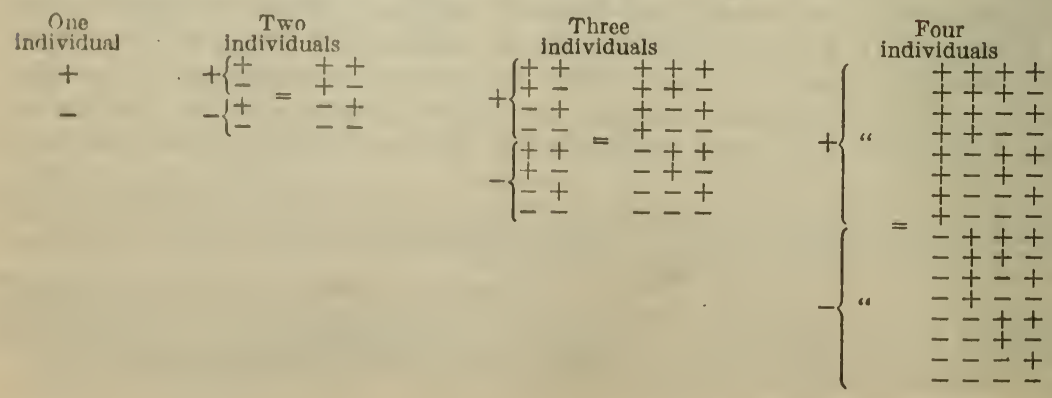
It follows immediately, from an enumeration of the various possibilities in the above representation, that with two animals the chance of zero mortality is 0.25 , the chance of 50 per cent mortality is 0.50 , and the chance of 100 per cent mortality is 0.25 ; with three animals, mortalities of $0,33 \frac{1}{3}, 662 / 3$, and 100 per cent correspond, respectively, to probabilities $0.125,0.375,0.375$, and 0.125 . A graphical representation of these probabilities, among others, is given in Figure 2.
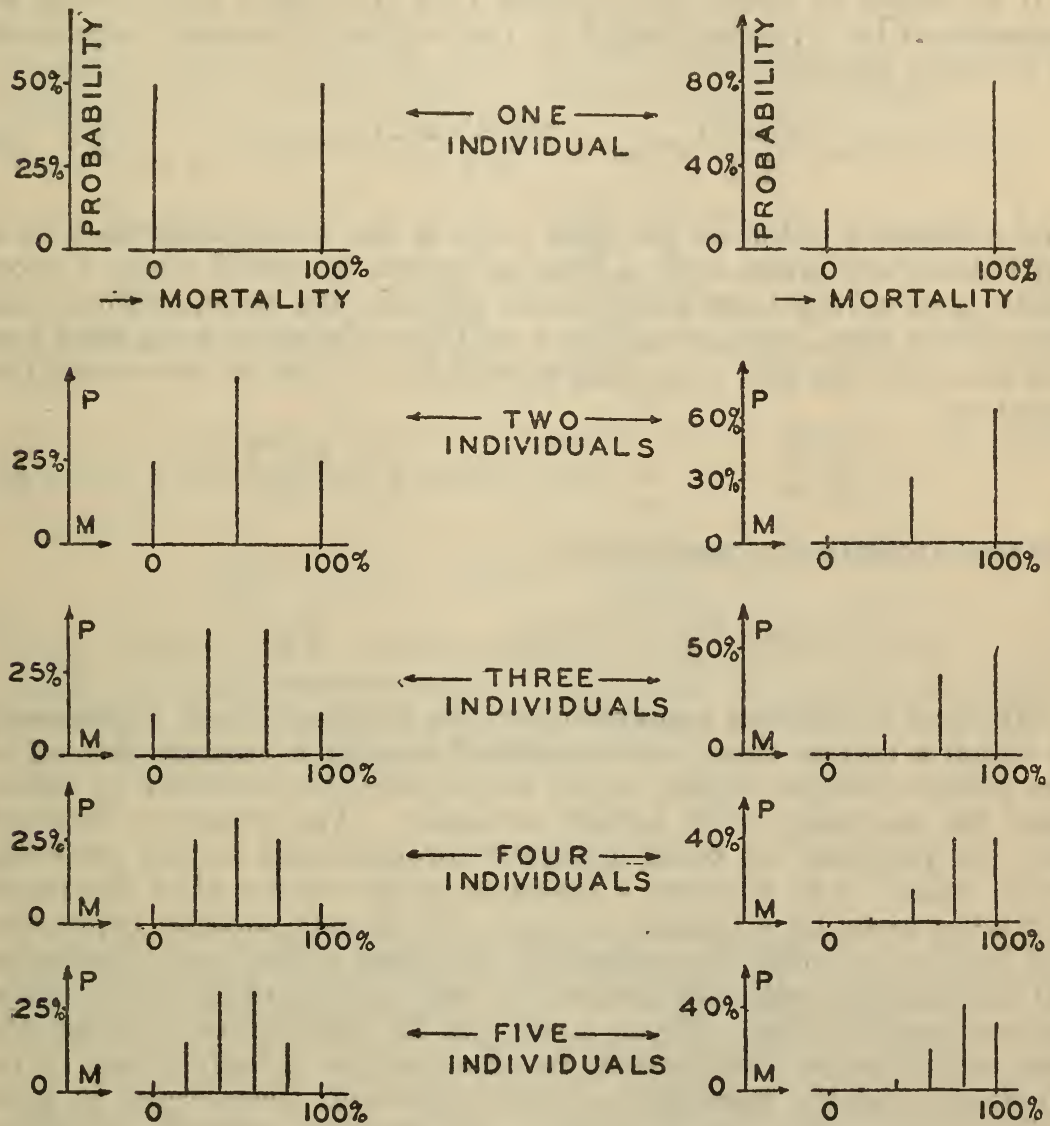

(a)

(b)

FIGURE 2.-Some probabilities for mortality in small groups

Probabilities of obtaining various values for mortality in experiments on small numbers of individuals $(a)$ for doses of poison which would kill 50 per cent of a very large group of individuals, and $(b)$ for doses which would produce 80 per cent mortality in a large group.

Figure $2(a)$ represents the case for a dose $D_{o}$, as was illustrated above, and Figure $2(b)$ gives the result for a larger dose of poison, one which would kill 80 per cent of the individuals in a large group. The method of getting results for dosages other than $D_{o}$ will be outlined below. It must be clearly understood that, although the above analysis signifies, for example, that if eight groups, with three individuals in a group, were given a dose of poison $D_{o}$, the most probable 
result would be that one group would entirely survive, one would entirely die, three groups would have only one death each, and three other groups would have only two deaths each, it does not signify that this result will always be obtained in practice; in fact it is one purpose of the present paper to find how in practice the chance of obtaining the most probable result varies with the number of individuals treated.

It is shown in books on statistics that the above results may be represented by a formula which is the ordinary binomial expansion of $(s+m)^{n}$, namely

$$
s^{n}+\frac{n}{1} s^{n-1} m+\frac{n(n-1)}{2 \cdot 1} s^{n-2} m^{2}+\frac{n(n-1)(n-2)}{3 \cdot 2 \cdot 1} s^{n-3} m^{3}+\ldots .+m^{n}
$$

The numerical value of the first term is the probability that, if $n$ individuals are given such a dose of poison as would cause a mortality $m$ in a very large group, none will die; the second term is the probability that only one of the $n$ will die; the third term that two will die, etc.; the last term that all will die. That is, for mortalities equal to

$$
0, \frac{1}{n}, \frac{2}{n} \ldots \ldots \ldots \frac{n-1}{n}, 1
$$

the probabilities are, respectively

$$
s^{n}, \frac{n}{1} s^{n-1} m, \frac{n(n-1)}{2 \cdot 1} s^{n-2} m^{2} \ldots \ldots \frac{n}{1} s m^{n-1}, m^{n}
$$

We next require an expression for the accuracy of an experiment in which a certain dose is administered to only $n$ animals instead of the infinite number which would be required theoretically to determine the mortality with perfect accuracy. The accuracy obtained may be regarded as being inversely proportional to the probable error, which is, in a sense, a measure of the chance that the result will be in error by a certain amount. Obviously such an expression will involve not only the probability for given errors in $m$ (expression (4) above), but will also involve the errors in $m$ obtained for these various probabilities. These errors are the differences between the true mortality $m$ which would be observed for a large group of individuals, and the mortality actually obtained; these errors in mortality corresponding, respectively, to the various terms in expression (4) are obviously obtained directly from equation (3), and are as follows:

$$
m, m-\frac{1}{n}, m-\frac{2}{n} \ldots \ldots \ldots m-\frac{n-1}{n}, m-1
$$

An expression for $\sigma$, the mean error in the mortality (or the "standard deviation" of statistics), in conformity with customary usage is the following:

$$
\sigma=\sqrt{\frac{\Sigma P(\Delta m)^{2}}{\Sigma P}}
$$

where $P$ represents a term of formula $(4)$ and $\Delta m$ represents the corresponding term of formula (5) and $\Sigma$ signifies that a summation is to 
be made over all terms of the formulas. Substituting from the formulas, we have

$$
\sigma^{2}=\frac{s^{n} m^{2}+\frac{n}{1} s^{n-1} m\left(m-\frac{1}{n}\right)^{2}+\frac{n(n-1)}{2 \cdot 1} s^{n-2} m^{2}\left(m-\frac{2}{n}\right)^{2}+\cdots+m^{n}\left(m-1^{2}\right)}{s^{n}+\frac{n}{1} s^{n-1} m+\frac{n(n-1)}{2 \cdot 1} s^{n-2} m^{2}+\frac{n(n-1)(n-2)}{3 \cdot 2 \cdot 1} s^{n-3} m^{3}+\cdots+m^{n}}
$$

or, expanding the squared terms in the numerator, and noting condensations that can be made by means of formula 2 above

$$
\begin{aligned}
& m^{2}(s+m)^{n} \\
&-2 m^{2}\left[s^{n-1}+\frac{n-1}{1} s^{n-2} m+\frac{(n-1)(n-2)}{2 \cdot 1} s^{n-3} m^{2}+\cdots+m^{n-1}\right] \\
& \sigma^{2}=+\frac{m}{n}\left[s^{n-1}+2 \frac{n-1}{1} s^{n-2} m+3 \frac{(n-1)(n-2)}{2 \cdot 1} s^{n-3} m^{2}+\cdots+n m^{n-1}\right] \\
&(s+m)^{n}
\end{aligned}
$$

Now we note that the first and second sets of terms in the square brackets above are equal, respectively, to

$$
\frac{1}{n} \frac{\partial}{\partial s}(s+m)^{n} \quad \text { and } \quad \frac{1}{n} \frac{\partial}{\partial m}\left[m \frac{\partial}{\partial s}(s+m)^{n}\right]
$$

this is easiest verified by differentiating the expanded form of $(s+m)^{n}$. Finally operating directly on the unexpanded binomial in the manner prescribed by formula (8), substituting in equation (7), reducing algebraically, and remembering that $s+m=1$, we readily find

$$
\sigma=\sqrt{\frac{s m}{n}}
$$

The above formula gives the mean error associated with the determination of any single point on the characteristic curve (that is, corresponding to some particular value of the dosage or poison or other effective agent, or to some particular concentration in which the organisms are placed, etc.), where $n$ is the number of individuals used in determining the point. The formula shows, for example, that if an equal number of individuals were used for each point, the mean error would be proportional to $\sqrt{s m}$; this quantity will be a maximum for $m=s=0.5$; that is

$$
\frac{d}{d m} \sqrt{s m}=\frac{d}{d m} \sqrt{m(1-m)}=1 / 2\left(m-m^{2}\right)^{-3 / 2}(1-2 m)=0, \text { or } m=0.5
$$

and will approach zero as either $m$ or $s$ approaches zero. This means that with equal numbers of individuals for all points, the points on the curve corresponding to values of $m$ close to 0 and to 1 , will have been obtained much more accurately than points for which $m$ is in the neighborhood of 0.5 . But for purposes of constructing a graph, there is usually no purpose in determining one point more accurately than any other; the accuracy of a graph may be ordinarily considered to 
be no greater than that of its least accurately determined region. Therefore, more individuals than necessary have been used to determine all the points except that for which $m=0.5$.

For a more efficient use of material, we may assume that, unless there happens to be some reason to the contrary, in a particular experiment, it is desired to obtain all parts of the characteristic curve with equal accuracy. Suppose the usual procedure has been adopted, wherein doses are given which are equally spaced on the axis of abscissæ ${ }^{6}$ then the above condition of equal accuracy can be met by distributing the individuals among the various points in such a fashion as to make the mean error associated with any point equal to that associated with any other point. That is, we wish to make $\sigma$ in equation (9) above equal to some constant, say $\left(4 n_{o}\right)^{-1 / 2}$

whence

$$
\sigma=\sqrt{\frac{s m}{n}}=\sqrt{\frac{1}{4 n_{0}}}
$$

$$
\frac{n}{n_{o}}=4 s m=4 m(1-m)=4 s(1-s)
$$

which appeared as equation (1) in the introduction. ${ }^{7}$

\section{TO DETERMINE THE DOSE REQUIRED TO PRODUCE A GIVEN MORTALITY}

If, as in many practical problems of biological assay, it is not required to determine the entire characteristic curve, but only the dose $D_{o}$ which will produce the end-point effect in a certain previously specified fraction $m_{0}\left(=1-s_{o}\right)$ of a group of individuals, a still more efficient use can be made of the available material. The method is dependent on the fact that, even if only a very small group of individuals is administered a dose of the effective agent, and the survival found, a most probable value for $D_{o}$ can be calculated at once from the characteristic curve ${ }^{8}$ and also the probable error associated with this result. If this probable error is too large, a different dose (and the method specifies what will be best to use next) may be administered to another small group, and again the most probable value for $D_{o}$ (with associated probable error) calculated making best use of the results from both sets of animals used so far. If this second probable error is still too large, the process may be repeated as often as necessary, the progress of the experiment always being guided in the best manner by all data secured up to that stage, and the final result always making best use of all data and specifying the accuracy quantitatively.

- The proper construction of a scgment of a curvo from a set of observed points is in reality influenced by all the points in the immediate vicinity of the segment. Therefore, if the values for the dosage were grouped closer together in one region than in another, the curvo would ordinarily have been determined with greater aecuracy in the former region. A uniform distribution is chosen above for simplicity.

7 It might appear to have been better to rcplace $n$ by $n-1$ in these formulas, as is shown in the theory of errors to bo neccssary in a caleulation of mean error involving the arithmetic mean of the observations instead of the true value of the quantlty to be determined (that is, corresponding to $d$ posteriori probabilities). However, it is hore supposed that tho mean errors for the various observed points (and thus the optimum distribution of material) are calculated from tho smoothed charaeteristic curve (or its proliminary determination). Here the ordinates for any region of the curve are really influenced by all determined points in the vioinity, and thus the value for $n$ which is effective in this regard is much greater than the value of $n$ used for any one polnt ou the curve, and the fractional differcnee between $n$ and $n-1$ becomes negligible.

In ordinary routine assay work, the shape of the curve is known accurately, and it is merely desired to And the potency of a given preparation; this corresponds to determining the scale of abscissae for that jreparation. When the curve is not known, the above method may still be of considerable value if a reasonable shape for tho curve bo assumed. 
In order to describe the calculations required above, let us consider any one, say $n_{k}$, of the small groups of individuals, $n_{a}, n_{b}, n_{c} \ldots \ldots$ which may be used in the experiment: Suppose a survival $s_{k}$ is found for this group when a dosage $D_{K}$ is administered to each individual. So far as can be determined from only this single isolated part of the experiment, $s_{k}$ is the most probable value of the ordinate on the characteristic curve corresponding to abscissa $D_{K}$, and the most probable value of $D_{o}$, say $D_{k}$, is found by simply noting the position of $s_{k}$ on the characteristic curve and multiplying $D_{K}$ by such a ratio as will appear to have produced a survival of $s_{o}$ instead of $s_{k}\left(s_{o}=1-m_{o}\right.$; see above). If the characteristic curve be represented by the relation $D=f(s)$, this calculation of $D_{k}$ may be described symbolically as follows:

$$
D_{k}=D_{K} \frac{f\left(s_{o}\right)}{f\left(s_{k}\right)}
$$

To find the probable error associated with $D_{k}$, we note first that the probable error associated with $s_{k}$ is

$$
\Delta s_{k}=0.6745 \sqrt{\frac{s_{k}\left(1-s_{k}\right)}{n_{k}-1}}
$$

this is the same as formula (10) except for a change in the denominator of the fraction as discussed in footnote 7 and the necessary change from mean to probable error. When $s$ varies along the characteristic curve from $s_{k}-\Delta s_{k}$ to $s_{k}+\Delta s_{k}, D$ obviously varies from $f\left(s_{k}-\Delta s_{k}\right)$ to $f\left(s_{k}+\Delta s_{k}\right)$; for the purposes of the present paper, ${ }^{9}$ the probable error $\Delta D_{k}$ to be associated with $D_{k}$ may be taken to be

$$
\frac{f\left(s_{o}\right)}{f\left(s_{k}\right)} \cdot \frac{f\left(s_{k}-\Delta s_{k}\right)-f\left(s_{k}+\Delta s_{k}\right)}{2}
$$

The fraction on the right above is the error associated with $D_{K}$, and the purpose of the fraction on the left is simply to cause $D_{k}$ to have the same percentage error as $D_{K}$.

Now, to begin the experiment, an arbitrary dose $D_{A}$ is given each individual in a small group, $n_{a}$, the survival, $s_{a}$, is noted, and the probable value, $D_{a}$, of $D_{o}$ with its associated error, $\Delta D_{a}$, is calculated as above: ${ }^{10}$ (For additional clarity, an example of these calculations is given in fig. 3.) If $\Delta D_{a}$ is too large, we give each individual of a

- It may be pointed out that the method described by equation (13) for the calculation of $\Delta D_{k}$ from $\Delta s_{k}$ is only a special case of a more general method. For example, instead of finding $\Delta D_{k}$ from $f\left(s_{k}-\Delta s_{k}\right)$ and $f\left(s_{k}+\Delta s_{k}\right)$ we might have found $R \Delta D_{k}$ from $f\left(s_{k}-R \Delta s_{k}\right)$ and $f\left(s_{k}+R \Delta s_{k}\right)$, where $R$ is a constant the value of which is a matter of choice. When $R$ is relatively small, when $s_{k}$ lies on a relatively straight portion of the graph, and when the determination of $s_{k}$ is relatively accurate (that is, when $\Delta s_{k}$ is small), the value found for $\Delta D_{k}$ will not depend appreciably on $R$; on the other hand, when the graph is strongly curved, etc., $\Delta D_{k}$ depends on $R$. It may be pointed out further that when $s_{k}-R \Delta s_{k}$ is equal to or less than zero, or when $s_{k}+R \Delta s_{k}$ is equal to or greater than 1.0, $\Delta D_{k}$ becomes inflnite, and consequently tho result with the group of animals $n_{k}$ is discarded so far as its effect on the probable error of the final result is concerned. (Seo Equation (14).) Both of these features are believed to be desirable, and the choice $R=1$ seems a reasonable value to select, although for some purposes other values of $R$ may be found to be preferable.

10 The degree of success attained with the above method depends to some extent on the determination of the first dose to be given, $D_{A}$. For the closer $D_{A}$ happens to approximate $D_{0}$, the fewer individuals will be required to attain a certain accuracy of determination of $D_{o}$. For example, until a value for $D_{A}$ is found which gives $s_{a}$ a value different from either zero or 100 per cent (that is 1.0 ), no definite statement can be made about the most probable value of $D_{0}$; that is, mathematically, the value $D_{a}$ is indeterminate. However, the special case in which nothing whatever is known about the approximate magnitude of the dose required to produce a known end-point effect is seldom encountered in practice. When it is encountered, the method usually followed when animals are valuable is to administer gradually increasing doses to a single animal, making some kind of allowance for a possible adaptation. 
second group, $n_{b}$, a dose $D_{B}=D_{a}$, and as before find $D_{b}$ and $\Delta D_{b}$. As is shown in the theory of errors, we may combine the results of the two experiments, and find that the most probable value for $D_{0}$ is now

$$
D_{C}=\frac{D_{a} /\left(\Delta D_{a}\right)^{2}+D_{b} /\left(\Delta D_{b}\right)^{2}}{1 /\left(\Delta D_{a}\right)^{2}+1 /\left(\Delta D_{b}\right)^{2}}
$$

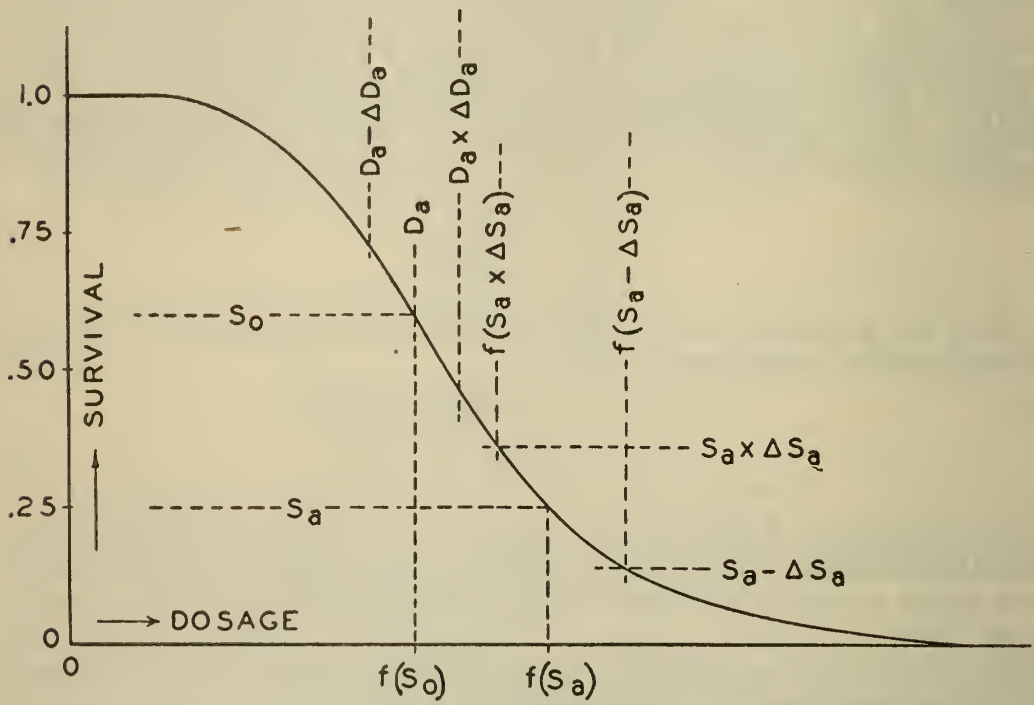

FIGURE 3.-An example of the assay of a preparation of unknown strength, but for which the shape of the characteristic curve is known

The strength of a preparation can be described by specifying the dose $D_{0}$ which would be required to produce its characteristic effect in a fraction $s_{0}$ (say for example $s_{0}=0.60$ ) of a large number of animals. Suppose $\mathrm{a}$ dose $D_{\Delta}$ is administered to $n_{s}=8$ individuals and it is found that 2 do not register the characteristic effect (that is, a survival $s_{a}=0.25$ is observed). Then the most probable value $D_{a}$ for $D_{o}$ is $D_{\Delta}$ times the ratio of the abcissae which correspond to $s_{0}$ and $s_{a}$ (equation (11)); for example, in the present case $D_{a}=0.72 D$.

The probable error (equation 12 ) in $s_{a}$ is

$$
\Delta s_{a}=0.6745 \sqrt{\frac{0.25 \times 0.75}{8-1}}=0.110
$$

The values of dosage corresponding to the limits $s_{a}+\Delta s_{a}(=0.36)$ and $s_{a}-\Delta s_{a}(=0.14)$ are $f\left(s_{a}-\Delta s_{a}\right)=1.16 D_{\Delta}$ and $f\left(s_{a}+\Delta s_{a}\right)=0.90 D_{A}$, and the probable error associated with $D_{A}$ may be approximated with

$$
\frac{f\left(s_{a}-\Delta s_{a}\right)-f\left(s_{a}+\Delta s_{a}\right)}{2}\left(=0.13 D_{\mathcal{A}} \text { in the present case }\right)
$$

Likewise, the probable error in $D_{a}$ must be $0.13 D_{a}$, which is equal to $\frac{f\left(s_{0}\right)}{f\left(s_{a}\right)} \times 0.13 \quad D_{\Delta}=0.09 D_{\Delta}$ (compare equation (13)). Thus, finally, $D_{o}=(0.72 \pm 0.09) D_{A}$ for the case taken here. If this happens to be an undesirably large error, a second group of animals, $n_{b}$, may be administered dosages $D_{B}=0.72 D_{A}$, and so on, as described in the text. In ordinary assay work, where standard numbers of animals are used for $n_{a}, n_{b}$, etc., the above calculations will reduce to a very simple routine.

and that the probable error associated with this value is

$$
\frac{1}{\sqrt{\left.1 /\left(\Delta D_{a}\right)^{2}+1 / \Delta D_{b}\right)^{2}}}
$$

We can now give to a third group, $n_{c}$, dose $D_{C}$, and find $D_{c}$, etc., in a similar fashion. In general, after the $k$ th group, $n_{k}$, has been given 
dose $D_{K}$, and $D_{k}$ and $\Delta D_{k}$ have been found, the most probable value for $D_{o}$ will be

$$
\frac{D_{a} /\left(\Delta D_{a}\right)^{2}+D_{b} /\left(\Delta D_{b}\right)^{2}+D_{c} /\left(\Delta D_{c}\right)^{2}+\ldots \ldots+D_{k} /\left(\Delta D_{k}\right)^{2}}{1 /\left(\Delta D_{a}\right)^{2}+1 /\left(\Delta D_{b}\right)^{2}+1 /\left(\Delta D_{c}\right)^{2}+\ldots \ldots+1 /\left(\Delta D_{k}\right)^{2}}
$$

and the probable error associated with this value will be the reciprocal of the square root of the denominator of this expression (14).

I am very much indebted to Dr. Raymond Pearl for consultation and suggestions regarding the above analysis. Also I wish to acknowledge courtesies extended me by the Marine Biological Laboratory at Woods Hole during the preparation of the paper.

Washington, August 30, 1930. 